\title{
The Approximate Solution of Fredholm Integral Equations with Oscillatory Trigonometric Kernels
}

\author{
Qinghua Wu \\ School of Mathematics and Statistics, Central South University, Changsha, Hunan 410083, China \\ Correspondence should be addressed to Qinghua Wu; jackwqh@sina.com
}

Received 9 March 2014; Accepted 4 April 2014; Published 17 April 2014

Academic Editor: Turgut Öziş

Copyright ( 2014 Qinghua Wu. This is an open access article distributed under the Creative Commons Attribution License, which permits unrestricted use, distribution, and reproduction in any medium, provided the original work is properly cited.

A method for approximating the solution of weakly singular Fredholm integral equation of the second kind with highly oscillatory trigonometric kernel is presented. The unknown function is approximated by expansion of Chebychev polynomial and the coefficients are determinated by classical collocation method. Due to the highly oscillatory kernels of integral equation, the discretised collocation equation will give rise to the computation of oscillatory integrals. These integrals are calculated by using recursion formula derived from the fundamental recurrence relation of Chebyshev polynomial. The effectiveness and accuracy of the proposed method are tested by numerical examples.

\section{Introduction}

The Fredholm integral equations of second kind,

$$
\begin{array}{r}
y(x)+\lambda \int_{a}^{b} \frac{K(x, t)}{|x-t|^{\alpha}} y(t) d t=f(x), \\
x \in[a, b], \quad 0<\alpha<1,
\end{array}
$$

where $K(x, t)$ is a continuous function and $f(x)$ is a given function, have many applications in mathematical physics and engineering, such as heat conduction problem, potential problems, quantum mechanics, and seismology image processing [1-4]. Particularly, when $K(x, x) \neq 0$ and $0<\alpha<1$, (1) is called weakly singular.

In most of the cases, the integral equation cannot be done analytically and one has to resort to numerical methods. Many numerical methods, such as collocation method and Galerkin method, have been developed to solve (1); for details see $[2,5,6]$. These methods are well-established numerical algorithms; however, standard version of these classical methods may suffer from difficulty for computation of (1), containing highly oscillatory kernels since the computation of the highly oscillatory integrals by standard quadrature methods is exceedingly difficult and the cost steeply increases with the frequency. Furthermore, Galerkin method requires many double integrals when approximating the solution of integral equation. Specially, when the kernel is highly oscillatory, it requires the evaluation of many highly oscillatory double integrals, which can become computationally expensive.

Recently, for weakly singular Volterra integral equations of the second kind with highly oscillatory Bessel kernels, it was found that the collocation methods are much more easily implemented and can get higher accuracy than discontinuous Galerkin methods under the same piecewise polynomials space; for details see [7-10]. In addition, collocation method only involves single integrals which are a little easier to evaluate. Motivated by this fact, here we investigate the application of collocation method for the solution of Fredholm integral equation

$$
\begin{array}{r}
y(x)+\lambda \int_{a}^{b} \frac{e^{i \omega(x-t)}}{|x-t|^{\alpha}} y(t) d t=f(x), \\
x \in[a, b], \quad \alpha<1, \quad \omega \gg 1 .
\end{array}
$$

Here $y(x)$ is the unknown function and $f(x)$ is a given function and assume that $\lambda$ is not the eigenvalue of integral equation, since for any finite interval $[a, b]$ it can be transformed to interval $[-1,1]$ by linear transformation. In this paper, we only consider the case of $[a, b]=[-1,1]$.

On the other hand, when the unknown function is approximated by Chebychev polynomial, the discretization of 
the integral equation (2) by collocation method will give rise to highly oscillatory integral which can be formulated as

$$
\int_{a}^{b} \frac{e^{(i \omega(x-t))}}{|x-t|^{\alpha}} T_{j}(t) d x, \quad x \in[a, b], 0<\alpha<1,
$$

where $T_{j}(x)$ denotes the Chebychev polynomials of the first kind.

In last few years many efficient methods have been devised for the evaluation of oscillatory integral, such as asymptotic method [11], Filon-type method [12, 13], Levin's collocation method [14], modified Clenshaw-Curtis method, Clenshaw-Curtis-Filon-type method [15], and generalized quadrature rule [16], although some of these methods do not involve Chebyshev polynomial. Piessens and Poleunis [17] consider a simpler case of $\alpha=0$ and use Chebyshev polynomials to evaluate the integral by somewhat indirect method involving a truncated infinite series of Bessel functions. The evaluation of this infinite summation suggests a defect of this method. An alternative procedure which avoids this infinite series is the original Bakhvalo and Vasil'eva-Legendre work involved in the evaluation of the integral

$$
M_{i}(\omega)=\int_{-1}^{1} P_{i}(x) e^{i \omega x} d x,
$$

by recurrence relation, where $P_{i}(x)$ denotes Legendre polynomial. Sadly, this relation proved to be unstable in the forwards direction for small $\omega$. In [18], Alaylioglu et al proposed a simple alternative approach analogous to Newton-Cotes based formulae. This method avoids the instability to a certain degree compared with these two methods mentioned above. We used the same idea and generalized it to the case of weakly singular and calculated oscillatory integral (3).

This paper is organized as follows: in Section 2 we derive some basic formulae and introduce some mathematical preliminaries of the proposed method. In Section 3 we discuss the evaluation of the integrals occurring in collocation equation. In Section 4 numerical experiments are conducted to illustrate the performance of the proposed method.

\section{Fundamental Relations}

Firstly, by separation of real and imaginary part of $y(x)$ and $e^{i w(x-t)}$, we transform the integral equation (2) into equivalent systems of two linear integral equations of Fredholm in the forms

$$
\begin{aligned}
& y_{1}(x)+\lambda \int_{-1}^{1} \frac{\cos (\omega(x-t))}{|x-t|^{\alpha}} y_{1}(t) d t \\
& -\lambda \int_{-1}^{1} \frac{\sin (\omega(x-t))}{|x-t|^{\alpha}} y_{2}(t) d t=f_{1}(x), \\
& y_{2}(x)+\lambda \int_{-1}^{1} \frac{\sin (\omega(x-t))}{|x-t|^{\alpha}} y_{1}(t) d t \\
& +\lambda \int_{-1}^{1} \frac{\cos (\omega(x-t))}{|x-t|^{\alpha}} y_{2}(t) d t=f_{2}(x),
\end{aligned}
$$

where $y(x)=y_{1}(x)+i y_{2}(x), f(x)=f_{1}(x)+i f_{2}(x)$, and $i=$ $\sqrt{-1}$.
Assume that $\mathbf{y}(x)=\left(y_{1}(x), y_{2}(x)\right)^{T}$ and $\mathbf{f}(x)=\left(f_{1}(x)\right.$, $\left.f_{2}(x)\right)^{T}$; then, systems of linear integral equations (5) can be written in the matrix form

$$
\mathbf{I y}(x)+\lambda \int_{-1}^{1} \mathbf{K}(x, t) \mathbf{y}(t) d t=\mathbf{f}(x),
$$

where

$$
\begin{aligned}
& \mathbf{I}=\left(\begin{array}{ll}
1 & 0 \\
0 & 1
\end{array}\right), \\
& \mathbf{K}(x, t)=\left(\begin{array}{cc}
\frac{\cos (\omega(x-t))}{|x-t|^{\alpha}} & \frac{-\sin (\omega(x-t))}{|x-t|^{\alpha}} \\
\frac{\sin (\omega(x-t))}{|x-t|^{\alpha}} & \frac{\cos (\omega(x-t))}{|x-t|^{\alpha}}
\end{array}\right) .
\end{aligned}
$$

Set $y_{i}(x)=\mathbf{T}(x) \mathbf{b}_{i}, i=1,2$, where $\mathbf{T}(x)=\left[T_{0}(x)\right.$, $\left.T_{1}(x), \ldots, T_{N}(x)\right], \mathbf{b}_{i}=\left[b_{i 0}, b_{i 1}, \ldots, b_{i N}\right]^{T}$.

Hence, unknown functions can be expressed by

$$
\mathbf{y}(x)=\mathscr{T} B,
$$

where

$$
\begin{gathered}
\mathbf{y}(x)=\left(\begin{array}{l}
y_{1}(x) \\
y_{2}(x)
\end{array}\right), \quad \mathscr{T}(x)=\left(\begin{array}{cc}
\mathbf{T}(x) & 0 \\
0 & \mathbf{T}(x)
\end{array}\right), \\
B=\left(\begin{array}{l}
\mathbf{b}_{1} \\
\mathbf{b}_{2}
\end{array}\right) .
\end{gathered}
$$

Then, the aim is to find Chebyshev coefficients, that is, the matrix $B$. We first substitute the Chebyshev collocation points, which are defined by $x_{i}=\cos (i \pi / N), i=0, \ldots, N$, into (6) and then rearrange a new matrix form to determine $B$ :

$$
\mathscr{I} \mathscr{Y}+\lambda \mathscr{K}=\mathscr{F}
$$

in which $\mathscr{K}$ is the integral part of (6) and

$$
\begin{aligned}
& \mathscr{I}=\left(\begin{array}{cccc}
\mathbf{I} & \mathbf{0} & \cdots & \mathbf{0} \\
\mathbf{0} & \mathbf{I} & \cdots & \mathbf{0} \\
\vdots & \vdots & \ddots & \vdots \\
\mathbf{0} & \mathbf{0} & \cdots & \mathbf{I}
\end{array}\right), \quad \mathscr{Y}=\left(\begin{array}{c}
\mathbf{y}\left(x_{0}\right) \\
\mathbf{y}\left(x_{1}\right) \\
\vdots \\
\mathbf{y}\left(x_{N}\right)
\end{array}\right), \\
& \mathscr{F}=\left(\begin{array}{c}
\mathbf{f}\left(x_{0}\right) \\
\mathbf{f}\left(x_{1}\right) \\
\vdots \\
\mathbf{f}\left(x_{N}\right)
\end{array}\right), \quad \mathscr{K}=\left(\begin{array}{c}
\mathbf{I K}\left(x_{0}\right) \\
\mathbf{I K}\left(x_{1}\right) \\
\vdots \\
\mathbf{I K}\left(x_{N}\right)
\end{array}\right) .
\end{aligned}
$$

By substituting (8) into (10), the unknown coefficients can be easily computed from this linear algebraic equations and therefore we find the solution of integral equation (2).

\section{Evaluation of the Integral}

$$
I[\omega, \alpha, j, x]=\int_{-1}^{1}\left(e^{(i \omega(x-t))} /|x-t|^{\alpha}\right) T_{j}(t) d t
$$

The discretization of integral equation will lead to the calculation of integral $I[\omega, \alpha, j, x]$. In this section, we will derive the recursion formula to compute it efficiently from the fundamental recurrence relation of Chebyshev polynomial. 


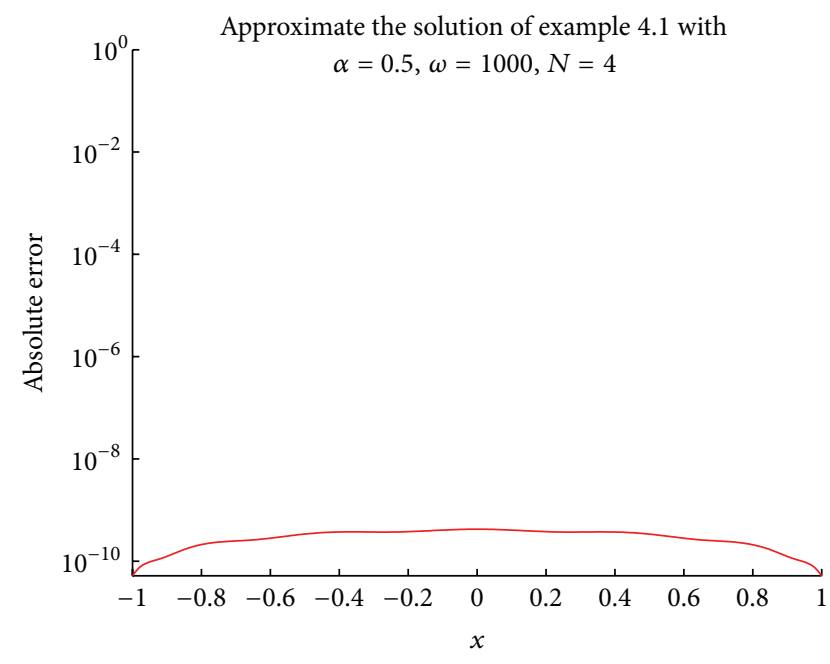

(a)

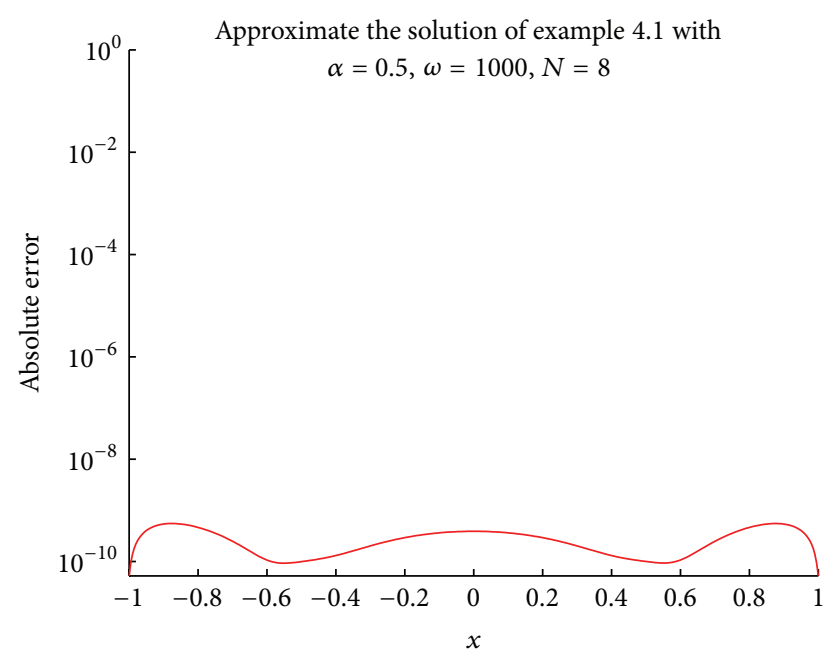

(b)

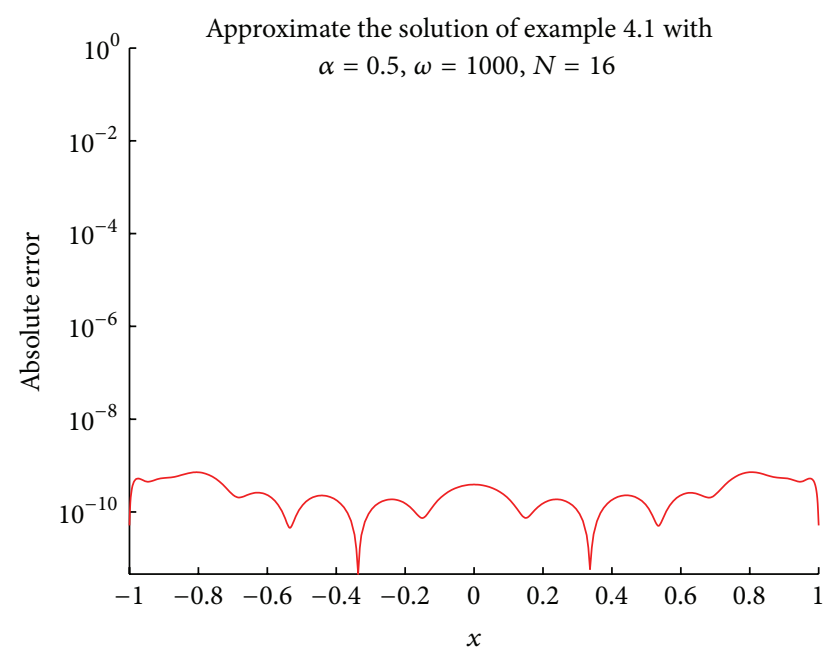

(c)

FIgURE 1: Example 1: the absolute error versus the $x$-coordinate for $N=4$ (a), $N=8$ (b), and $N=16$ (c) with negative data ignored.

The Chebyshev polynomials are of the form

$$
\begin{gathered}
T_{0}(x)=1 \\
T_{1}(x)=x \\
\vdots \\
T_{n}(x)=2 x T_{n-1}(x)-T_{n-2}(x), \quad n=2,3, \ldots,
\end{gathered}
$$

and the coefficients $C_{i, j}$ of $x^{j}$ in $T_{i}(x)$ can be easily calculated by the means of the recurrence relation

$$
C_{i, j}=2 C_{i-1, j-1}-C_{i-2, j}, \quad i \geq 2, \quad j \leq i .
$$

By expanding the Chebyshev polynomial $T_{i}(x)$ in terms of powers of $x$, the integral $I[\omega, \alpha, j, x]$ would be transformed into the form of

$$
I[\omega, \alpha, j, x]=\sum_{k=0}^{j} C_{j, k} I 1[\omega, \alpha, k, x],
$$

where $I 1[\omega, \alpha, k, x]=\int_{-1}^{1}\left(e^{(i \omega(x-t))} /|x-t|^{\alpha}\right) t^{k} d t$.
By separation of real and imaginary part of $e^{i w(x-t)},(14)$ is transformed into

$$
\begin{aligned}
I 1_{c}[\omega, \alpha, j, x] & =\int_{-1}^{1} \frac{t^{j}}{|x-t|^{\alpha}} \cos (\omega(x-t)) d t, \\
I 1_{s}[\omega, \alpha, j, x] & =\int_{-1}^{1} \frac{t^{j}}{|x-t|^{\alpha}} \sin (\omega(x-t)) d t,
\end{aligned}
$$

whose main difficulty now is turning to the evaluation of the following basic integrals:

$$
\begin{aligned}
& M 1_{c}[\omega, \alpha, j, x]=\int_{-1}^{1} \frac{t^{j}}{|x-t|^{\alpha}} \cos (\omega t) d t \\
& M 1_{s}[\omega, \alpha, j, x]=\int_{-1}^{1} \frac{t^{j}}{|x-t|^{\alpha}} \sin (\omega t) d t .
\end{aligned}
$$




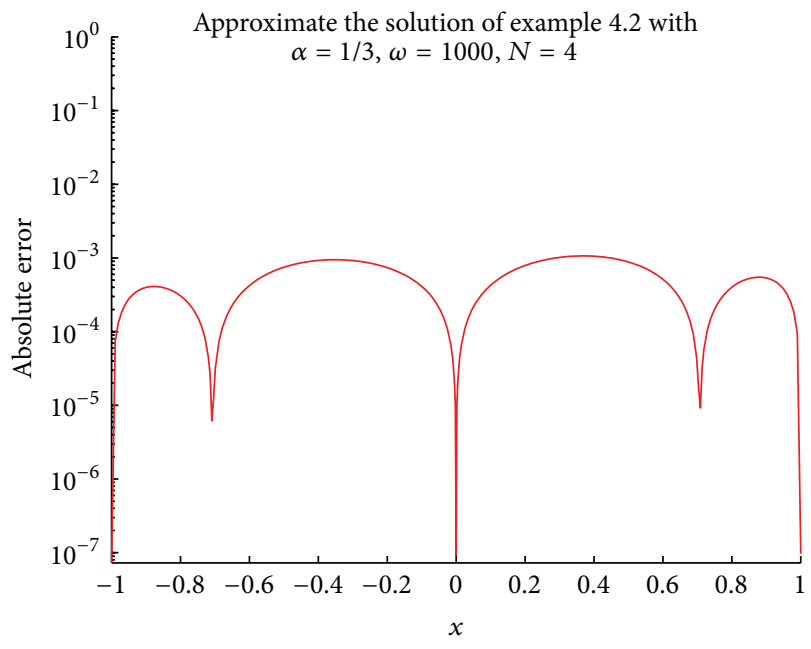

(a)

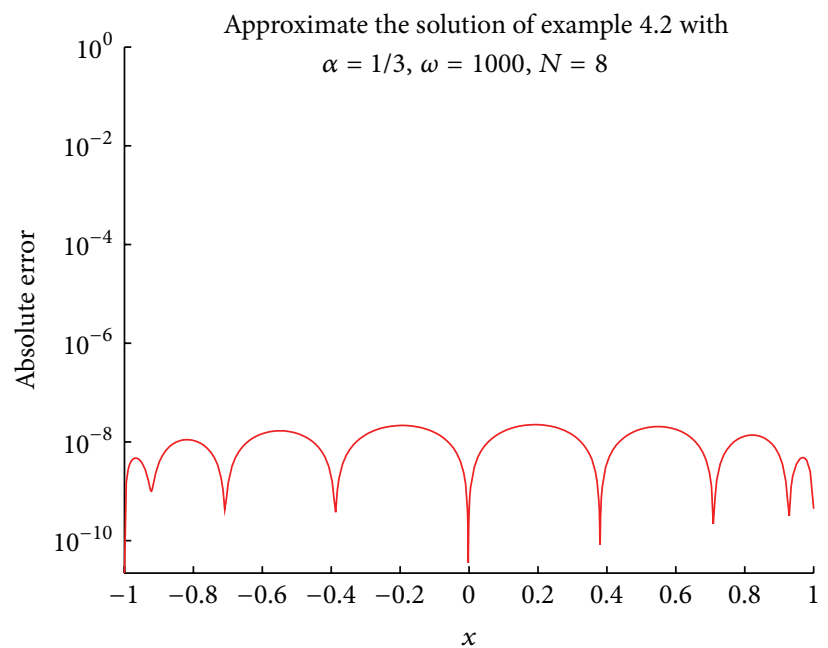

(b)

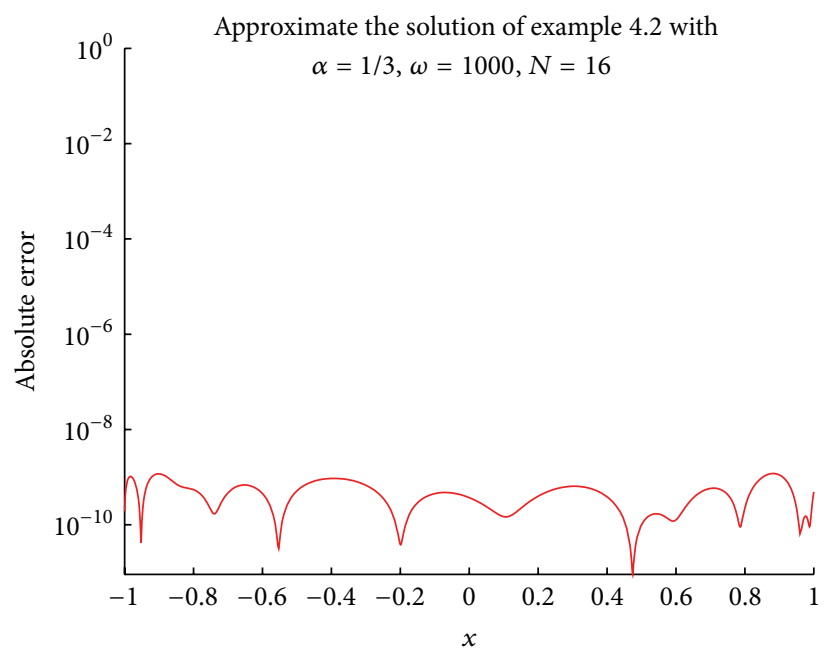

(c)

FIGURE 2: Example 2: the absolute error versus the $x$-coordinate for $N=4$ (a), $N=8$ (b), and $N=16$ (c) with negative data ignored.

With substitution for definite integral and binomial theorem, (16) is equivalent to

$$
\begin{aligned}
& M 1_{c}[\omega, \alpha, j, x] \\
&=\sum_{k=0}^{j} C_{j}^{k} x^{j-k}\left[(-1)^{k} \int_{0}^{x+1} t^{k-\alpha} \cos (\omega(x-t)) d t\right. \\
&\left.\quad+\int_{0}^{1-x} t^{k-\alpha} \cos (\omega(x-t)) d t\right],
\end{aligned}
$$

where $C_{j}^{k}$ is number of combination and $M 1_{s}[\omega, \alpha, j, x]$ can be formulated analogy.

Efficient evaluation of (18) is based on accurate calculation of integrals in the formula which can be computed explicitly by the incomplete Gamma function $\Gamma(z, \alpha)[19]$; that is,

$$
\begin{aligned}
& \int t^{\mu-1} \cos (\omega t) d t \\
& =\frac{1}{2}\left[(i \omega)^{-\mu} \Gamma(\mu, i \omega t)+(-i \omega)^{-\mu} \Gamma(\mu,-i \omega t)\right], \\
& \int t^{\mu-1} \sin (\omega t) d t \\
& =\frac{i}{2}\left[(i \omega)^{-\mu} \Gamma(\mu, i \omega t)-(-i \omega)^{-\mu} \Gamma(\mu,-i \omega t)\right],
\end{aligned}
$$

in which $\mu>0, x>0$; for details see ([20], pp 215).

Once the integral $I[\omega, \alpha, j, x]$ is obtained, by substituting into (10), the coefficient matrix is derived; then, we can compute the solution of integral by solving these linear algebraic equations. 
TABLE 1: Approximations for $y(x)+\int_{-1}^{1}\left(e^{i \omega(x-t)} /|x-t|^{0.5}\right) y(t) d t=e^{x}$ with $\omega=10^{3}$.

\begin{tabular}{lccc}
\hline$x$ & $\cos (\pi)$ & $\cos (3 \pi / 4)$ & $\cos (0)$ \\
\hline$\widehat{y}_{1}^{4}$ & 0.351696035495271 & 0.459037927481918 & 0.924223909091303 \\
$\widehat{y}_{1}^{8}$ & 0.351696947581043 & 0.459033811940511 & 0.924224547405338 \\
$\widehat{y}_{1}^{16}$ & 0.351741610883315 & 0.459036527929146 & 0.924226969620880 \\
$\widehat{y}_{2}^{4}$ & 0.0141286409694892 & 0.000146310591227839 & -0.00107727880701281 \\
$\widehat{y}_{2}^{8}$ & 0.0141193392298137 & 0.000145019893750479 & -0.00107593754108643 \\
$\widehat{y}_{2}^{16}$ & 0.0140583682955762 & 0.000144178555797097 & -0.00106881635211410 \\
\hline
\end{tabular}

TABLE 2: Approximations for $y(x)+\int_{-1}^{1}\left(e^{i \omega(x-t)} /|x-t|^{0.5}\right) y(t) d t=e^{x}$ with $\omega=10^{3}$.

\begin{tabular}{lcr}
\hline$x$ & $\cos (\pi / 4)$ & 1 \\
\hline$\hat{y}_{1}^{4}$ & 1.88249404678421 & 2.61059423521207 \\
$\widehat{y}_{1}^{8}$ & 1.88248275737555 & 2.61059120470589 \\
$\widehat{y}_{1}^{16}$ & 1.88248361661370 & 2.61073031676337 \\
$\widehat{y}_{2}^{4}$ & 0.00323202027752064 & -0.0995508315886191 \\
$\widehat{y}_{2}^{8}$ & 0.00321634589900894 & -0.0994917961267987 \\
$\widehat{y}_{2}^{16}$ & 0.00319500282444326 & -0.0993143176430846 \\
\hline
\end{tabular}

\section{Numerical Examples}

In this section, we give some numerical examples to illustrate the performance of proposed method. In all the following examples, $N+1$ is the number of mesh points, $\hat{y}_{i}^{N}(x)$ denotes the approximate solution, where $N$ is the number of terms of the Chebyshev series, and $y(x)$ denotes the exact solution, respectively. All the computations have been performed by using Matlab R2012a on a $2.5 \mathrm{GHz}$ PC with $2 \mathrm{~GB}$ of RAM.

Example 1. We consider $y(x)+\int_{-1}^{1}\left(e^{i \omega(x-t)} /|x-t|^{\alpha}\right) y(t) d t=$ $f(x)$ and set $f(x)=1+\int_{-1}^{1}\left(e^{i \omega(x-t)} /|x-t|^{\alpha}\right) d t$; then, the solution of which is

$$
y(x)=1
$$

We plot the absolute error $|y(x)-\hat{y}(x)|$ by Matlab 2012a internal function ezplot. Specially, for $N=4$, with $\omega=10^{3}$ and $\alpha=0.5$, solve linear algebraic equations (10); it can be found that the approximate solutions are

$$
\begin{aligned}
\widehat{y}_{1}(x)= & 0.9999999996-0.110 \times 10^{-15} x+0.281 \times 10^{-9} x^{2} \\
& +0.323 \times 10^{-15} x^{3}+0.563 \times 10^{-10} x^{4}, \\
\widehat{y}_{2}(x)= & 0.460 \times 10^{-14}-0.329 \times 10^{-9} x+0.554 \times 10^{-11} x^{2} \\
& +0.322 \times 10^{-9} x^{3}-0.555 \times 10^{-11} x^{4} .
\end{aligned}
$$

It is easy to see from Figure 1 that the proposed method is converging and we only need $N=4$ so we could achieve high accuracy.
Example 2. We consider $y(x)+\int_{-1}^{1}\left(e^{i \omega(x-t)} /|x-t|^{\alpha}\right) y(t) d t=$ $f(x)$ and set $f(x)=e^{x}+\int_{-1}^{1}\left(e^{i \omega(x-t)} /|x-t|^{\alpha}\right) e^{t} d t$; then, the solution of which is

$$
y(x)=e^{x} .
$$

In this case, for $N=4$, with $\omega=1000$ and $\alpha=1 / 3$, the approximate solutions are

$$
\begin{aligned}
\hat{y}_{1}(x)= & 1.000000001+0.9956819990 x+0.4992866904 x^{2} \\
& +0.1795192648 x^{3}+0.04379395375 x^{4}, \\
\hat{y}_{2}(x)= & -0.530 \times 10^{-7}+0.189 \times 10^{-7} x+0.426 \times 10^{-6} x^{2} \\
& -0.246 \times 10^{-7} x^{3}-0.420 \times 10^{-6} x^{4} .
\end{aligned}
$$

It is obvious to see from Figure 2 that the proposed method is efficient and could achieve high accuracy with little number of collocation points.

Example 3. For a general case, we consider $y(x)+\int_{-1}^{1}\left(e^{i \omega(x-t)} /|x-t|^{\alpha}\right) y(t) d t=e^{x}$ and set $\alpha=0.5$. Although the solution of this equation is unknown, we can verify the calculation precision of the method to a certain degree by comparing the approximate evaluation at mesh points.

In this case, for $N=4$, with $\omega=1000$ and $\alpha=0.5$, the approximate solutions are

$$
\begin{aligned}
\widehat{y}_{1}(x)= & 0.9242239091 \\
& +0.8836218495 x+0.4292470859 x^{2} \\
& +0.2458272504 x^{3}+0.1276741404 x^{4},
\end{aligned}
$$


TABLE 3: Approximations for $y(x)+\int_{-1}^{1}\left(e^{i \omega(x-t)} /|x-t|^{0.5}\right) y(t) d t=e^{x}$ with $\omega=10^{6}$.

\begin{tabular}{lccr}
\hline$x$ & $\cos (\pi)$ & $\cos (3 \pi / 4)$ & $\cos (0)$ \\
\hline$\widehat{y}_{1}^{4}$ & 0.367419631536920 & 0.491835233010361 & 0.997500711679274 \\
$\widehat{y}_{1}^{8}$ & 0.367419631541538 & 0.491835233006225 & 0.997500711679218 \\
$\widehat{y}_{1}^{16}$ & 0.367419632527633 & 0.491835233064998 & 0.997500711678921 \\
$\widehat{y}_{2}^{4}$ & 0.000458466865252363 & $0.150404190 \times 10^{-5}$ & $-0.219327280 \times 10^{-5}$ \\
$\widehat{y}_{2}^{8}$ & 0.000458466860662437 & $0.150403636 \times 10^{-5}$ & $-0.219326739 \times 10^{-5}$ \\
$\widehat{y}_{2}^{16}$ & 0.000458463698666909 & $0.150390826 \times 10^{-5}$ & $-0.219326736 \times 10^{-5}$ \\
\hline
\end{tabular}

TABLE 4: Approximations for $y(x)+\int_{-1}^{1}\left(e^{i \omega(x-t)} /|x-t|^{0.5}\right) y(t) d t=e^{x}$ with $\omega=10^{6}$.

\begin{tabular}{lcc}
\hline$x$ & $\cos (\pi / 4)$ & 1 \\
\hline$\widehat{y}_{1}^{4}$ & 2.02304108762491 & 2.71487514654707 \\
$\widehat{y}_{1}^{8}$ & 2.02304108760947 & 2.71487514654071 \\
$\widehat{y}_{1}^{16}$ & 2.02304108780835 & 2.71487515460854 \\
$\widehat{y}_{2}^{4}$ & $0.384042582 \times 10^{-5}$ & -0.00339813438891857 \\
$\widehat{y}_{2}^{8}$ & $0.384040621 \times 10^{-5}$ & -0.00339813431478428 \\
$\widehat{y}_{2}^{16}$ & $0.3840356665 \times 10^{-5}$ & -0.00339813322237100 \\
\hline
\end{tabular}

$$
\begin{aligned}
\widehat{y}_{2}(x)= & -0.001077278807+0.06120358877 x \\
& +0.05269959347 x^{2}-0.1180433250 x^{3} \\
& -0.09433340997 x^{4}
\end{aligned}
$$

It is also easy to see from Tables $1,2,3$, and 4 that the presented method is efficient and accurate, although the exact solution is unknown.

\section{Conclusion}

In this paper, we explore quadrature methods for weakly singular Fredholm integral equation of the second kind with oscillatory trigonometric kernels and present collocation methods with Chebyshev series for calculation of the solution. For integral equation with highly oscillatory kernels, the standard collocation methods with classical quadrature methods are not suitable for the numerical approximation of the solution of integral equation, since the computation of the highly oscillatory integrals by standard quadrature methods is exceedingly difficult and the cost steeply increases with the frequency. Based on the recursion formula derived in Section 3, we compute the highly oscillatory integrals occurring in collocation equation, directly and efficiently. Numerical examples demonstrate the performance of algorithm.

\section{Conflict of Interests}

The author declares that there is no conflict of interests regarding the publication of this paper.

\section{Acknowledgment}

This paper was supported by NSF of China (no. 11371376).

\section{References}

[1] V. M. Aleksandrov and E. V. Kovalenko, "Mathematical method in the displacement problem," Inzhenernyj Zhurnal. Mekhanika Tverdogo Tela, vol. 2, pp. 77-89, 1984.

[2] H. Brunner, On the Numerical Solution of First-Kind Volterra Integral Equations with Highly Oscillatory Kernels, Highly Oscillatory Problems: From Theory to Applications, Isaac Newton Institute, HOP 13-17, 2010.

[3] R. Estrada and R. P. Kanwal, Singular Integral Equations, Birkhäauser, Boston, Mass, USA, 2000.

[4] A. D. Polyanin and A. V. Manzhirov, Handbook of Integral Equations, CRC Press, 1998.

[5] H. Brunner, Collocation Methods for Volterra Integral and Related Functional Equations, Cambridge University Press, Cambridge, Cambridge, UK, 2004.

[6] I. G. Graham, "Galerkin methods for second kind integral equations with singularities," Mathematics of Computation, vol. 39, no. 160, pp. 519-533, 1982.

[7] S. Xiang and H. Brunner, "Efficient methods for Volterra integral equations with highly oscillatory Bessel kernels," BIT. Numerical Mathematics, vol. 53, no. 1, pp. 241-263, 2013.

[8] S. Xiang and K. He, "On the implementation of discontinuous Galerkin methods for Volterra integral equations with highly oscillatory Bessel kernels," Applied Mathematics and Computation, vol. 219, no. 9, pp. 4884-4891, 2013.

[9] S. Xiang and Q. Wu, "Numerical solutions to Volterra integral equations of the second kind with oscillatory trigonometric kernels," Applied Mathematics and Computation, vol. 223, pp. 34-44, 2013.

[10] Q. Wu, "On graded meshes for weakly singular Volterra integral equations with oscillatory trigonometric kernels," Journal of 
Computational and Applied Mathematics, vol. 263, pp. 370-376, 2014.

[11] A. Iserles and S. P. Nørsett, "On quadrature methods for highly oscillatory integrals and their implementation," BIT. Numerical Mathematics, vol. 44, no. 4, pp. 755-772, 2004.

[12] A. Iserles and S. P. Nørsett, "Efficient quadrature of highly oscillatory integrals using derivatives," Proceedings of The Royal Society of London A, vol. 461, no. 2057, pp. 1383-1399, 2005.

[13] S. Xiang, "Efficient Filon-type methods for $\int_{a}^{b} f(x) e^{i \omega g(x)} d x$," Numerische Mathematik, vol. 105, no. 4, pp. 633-658, 2007.

[14] D. Levin, "Fast integration of rapidly oscillatory functions," Journal of Computational and Applied Mathematics, vol. 67, no. 1, pp. 95-101, 1996.

[15] S. Xiang, Y. J. Cho, H. Wang, and H. Brunner, "ClenshawCurtis-Filon-type methods for highly oscillatory Bessel transforms and applications," IMA Journal of Numerical Analysis, vol. 31, no. 4, pp. 1281-1314, 2011.

[16] G. A. Evans and K. C. Chung, "Some theoretical aspects of generalised quadrature methods," Journal of Complexity, vol. 19, no. 3, pp. 272-285, 2003.

[17] R. Piessens and F. Poleunis, "A numerical method for the integration of oscillatory functions," vol. 11, pp. 317-327, 1971.

[18] A. Alaylioglu, G. A. Evans, and J. Hyslop, "The use of Chebyshev series for the evaluation of oscillatory integrals," The Computer Journal, vol. 19, no. 3, pp. 258-267, 1976.

[19] M. Abramowitz and I. A. Stegun, Handbook of Mathematical Functions, National Bureau of Standards, Washington, DC, USA, 1964.

[20] I. S. Gradshteyn and I. M. Ryzhik, Table of Integrals, Series, and Products, New York Academic Press, 1965. 


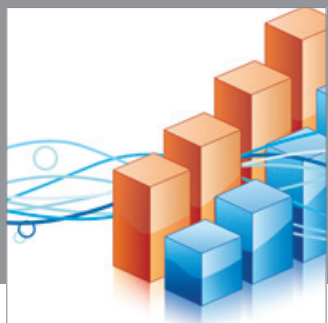

Advances in

Operations Research

mansans

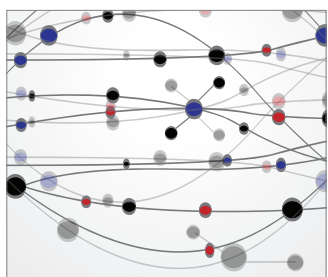

The Scientific World Journal
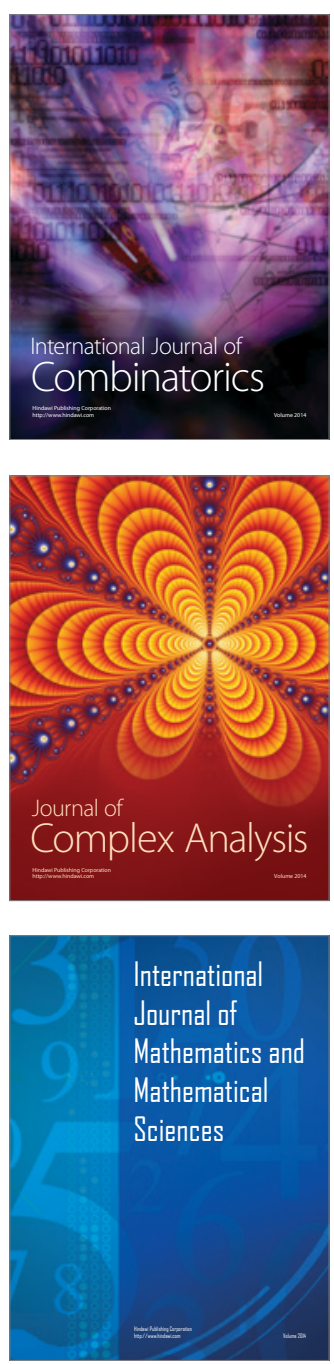
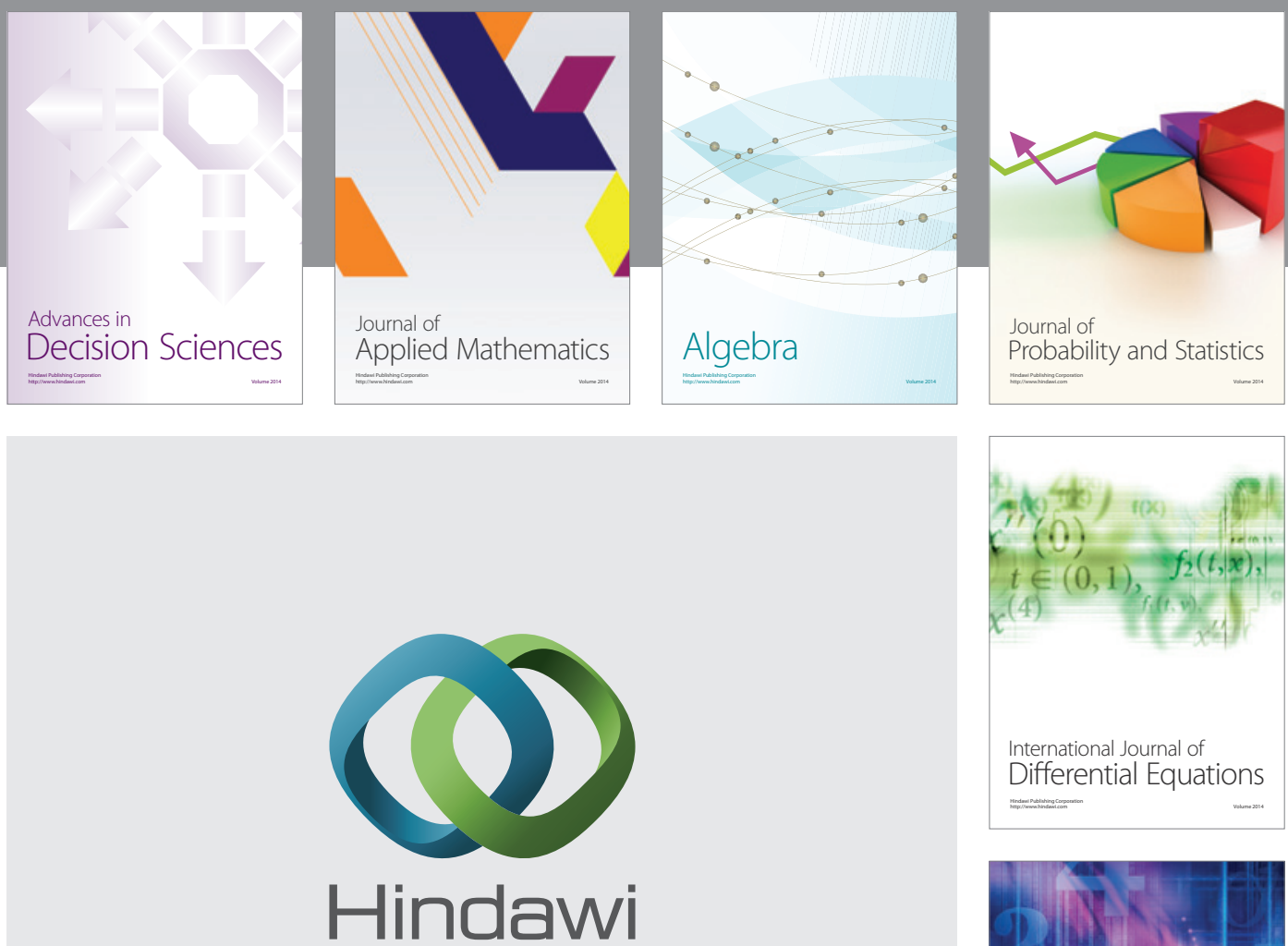

Submit your manuscripts at http://www.hindawi.com
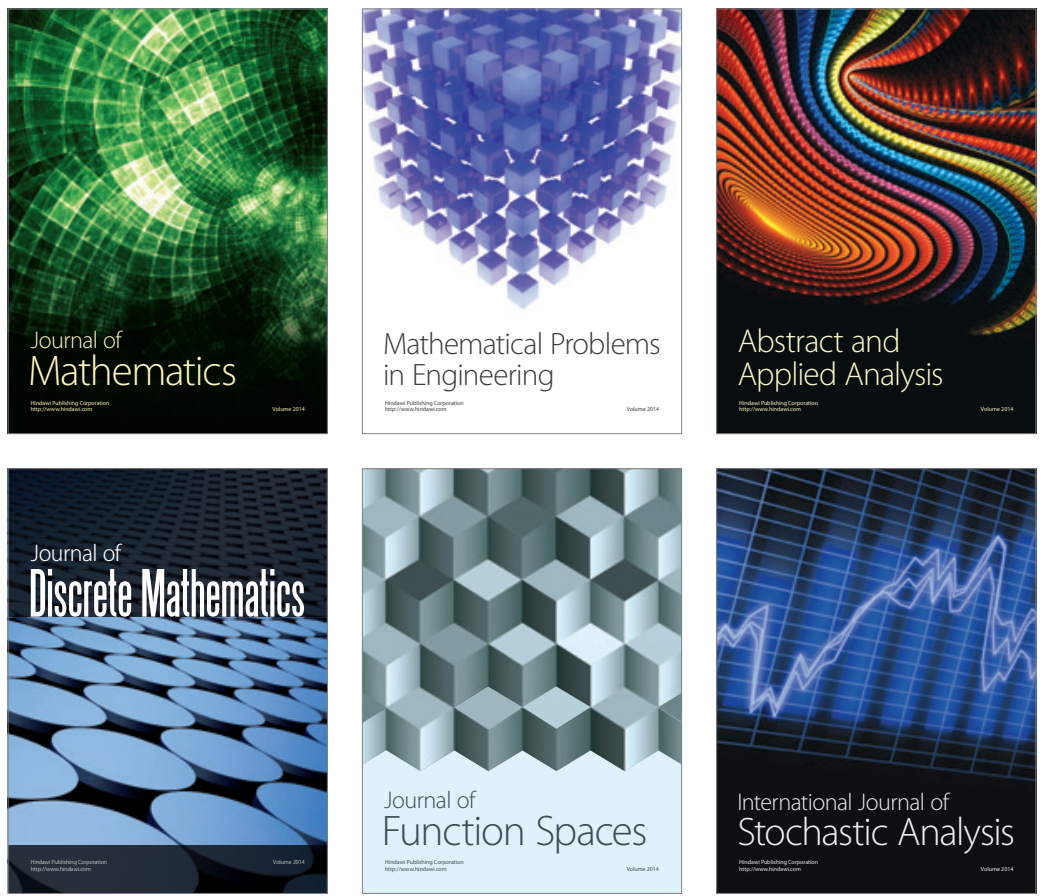

Journal of

Function Spaces

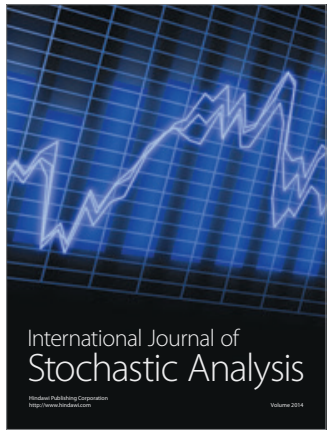

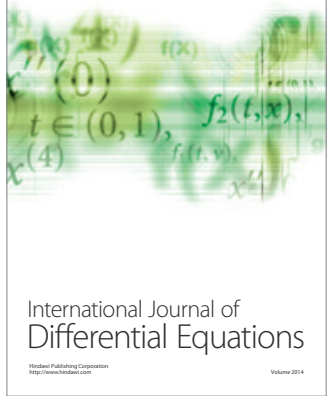
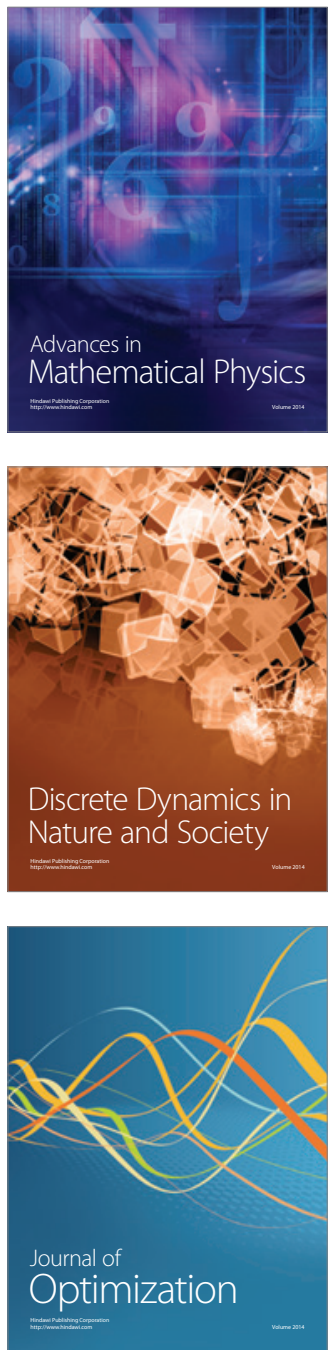